
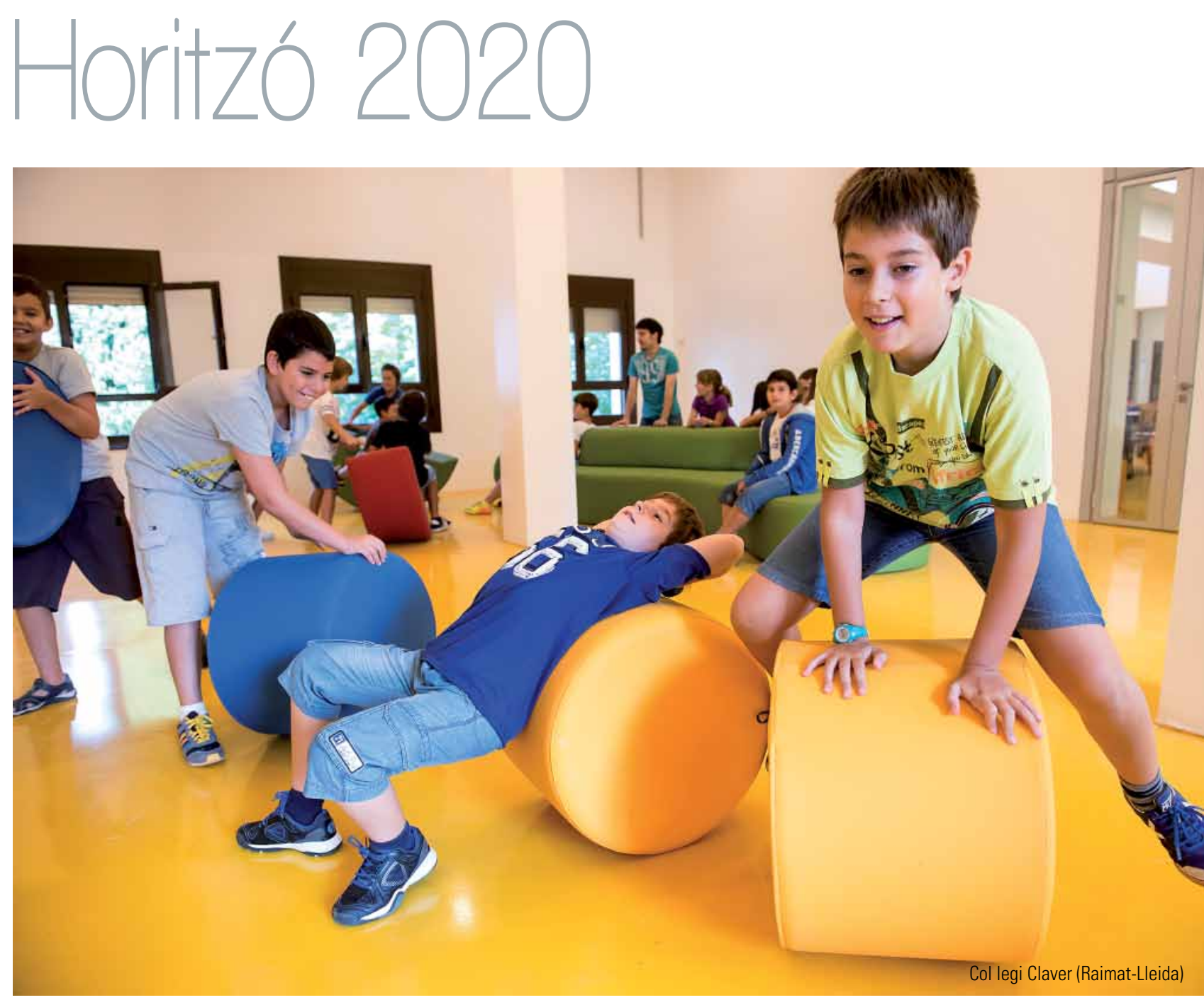

\title{
Crear una nueva escuela y hacerlo todos juntos
}

Los colegios jesuitas de Cataluña llevan a cabo una experiencia que es mucho más que una renovación de las técnicas pedagógicas en las escuelas, una mejora de la acción pastoral o un conjunto de pequeños cambios. Se trata de una trasformación

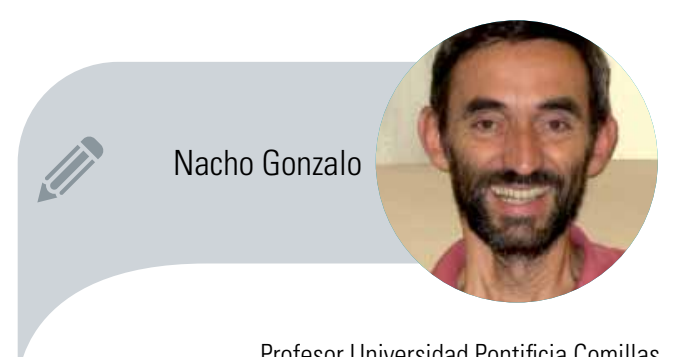

Profesor Universidad Pontificia Comillas igonzalo@comillas.edu profunda que haga sostenibles estas escuelas, que dé sentido a la acción educativa y que sea ejemplar en la concreción del compromiso evangélico de la Compañía de Jesús. 
Llevo unos días sumergido en los textos, los vídeos y otros materiales, de una experiencia de la que había oído hablar hace muchos meses, un runrún aquí y allá en ambientes educativos: los colegios jesuitas de Cataluña están metidos en unos trabajos de renovación pedagógica muy interesantes. Efectivamente, te pones a buscar y enseguida lo encuentras: "crear una nueva escuela y hacerlo todos juntos", decían en el año 2013, una nueva escuela que desarrolla y pone en práctica un nuevo modelo pedagógico, la Ratio Studiorum del siglo XXI, jcasi nada! Y tiene un nombre: Horitzó 2020, horizonte 2020.

Durante estos días han resonado en mi cabeza con fuerza las palabras del gran educador argentino Juan Carlos Tedesco, que nos habla del "profesor como profesional colectivo". Y resuenan por dos motivos. En primer lugar, por su concepción del papel del docente, pues constata que no está presente en las reformas educativas. Con frecuencia se toman en ellas decisiones sobre planes de estudio, horarios, textos, equipamiento y otros materiales, sistemas de evaluación, logros deseados o instrumentos para medirlos, pero no se considera al profesor como un elemento clave. Así, lo que pasa en las aulas, la relación profesor-alumno, sigue siendo prácticamente igual a la que teníamos hace mucho tiempo. Por eso insiste en el paso de una cultura de desempeño individual del profesor, a una idea de profesionalismo colectivo, que implica a su vez tanto la responsabilidad colectiva como la existencia de un equipo autónomo. En el año 2001, nos decía en Lima que a mayor autonomía del equipo docente se obtiene mayor calidad de la educación que ofrece.

En segundo lugar, y más pertinente si cabe para sintonizar con el Horitzó 2020 y con sus fines últimos, Tedesco, que fue director de la Oficina Internacional de Educación de la UNESCO en Ginebra entre 1992 y 1997, escribía hace diez años a un grupo de alumnos de magisterio de Comillas e insistía en la equidad de la educación para todos:

\begin{abstract}
"Quiero hacerte llegar a ti y a tus compañeros de carrera un saludo muy fraterno y decirles que el futuro depende cada vez más de la calidad de la educación que seamos capaces de brindar a todos. Tenemos una gran responsabilidad y tratemos de poner nuestra capacidad científica al servicio de los valores éticos de justicia y equidad".
\end{abstract}

Así que sumergirse en el ambiente del Horitzó 2020 (aunque sea sin haberlo visto en directo, todo

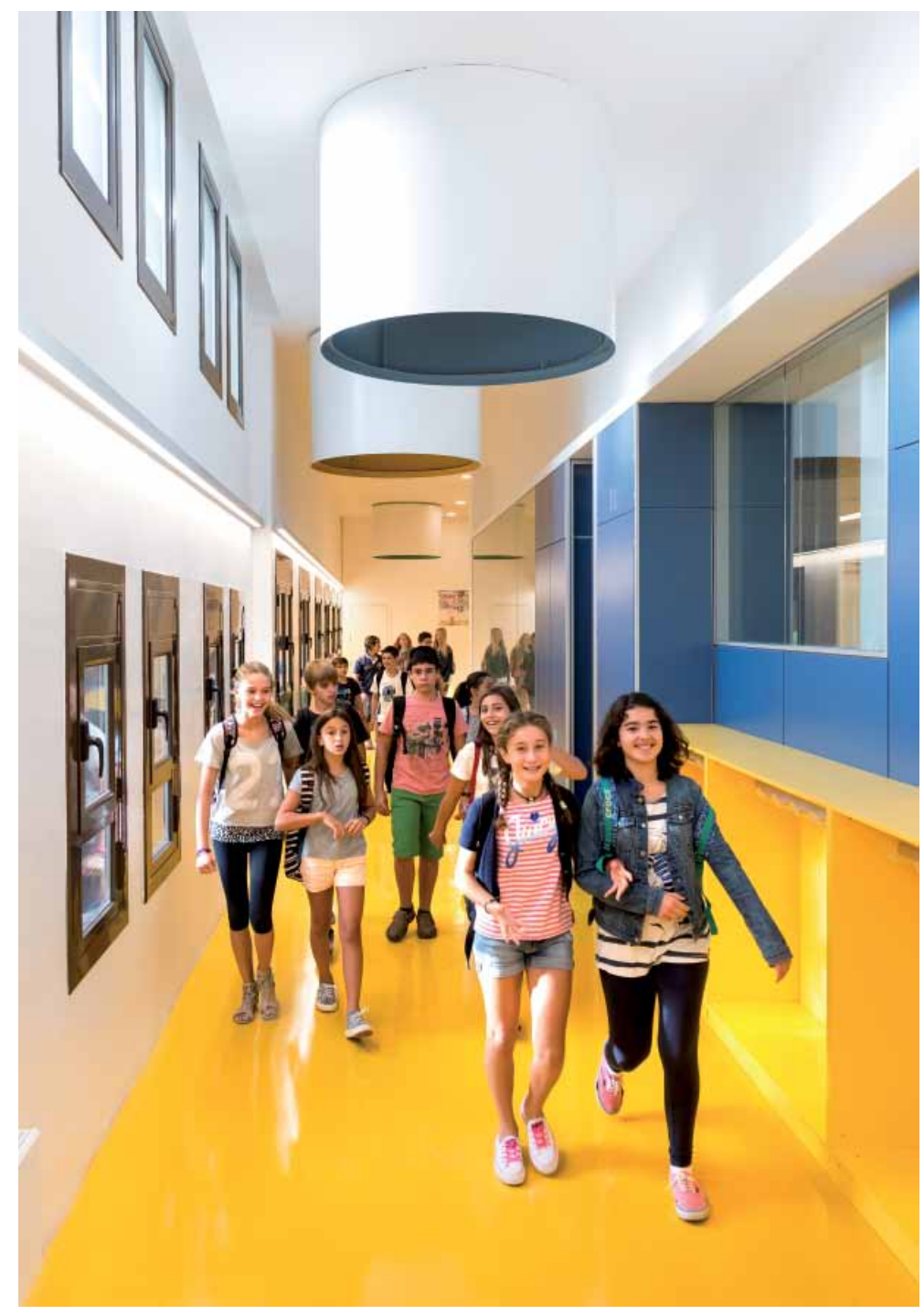

se andará) ha sido un continuo trastear con los valores de la pedagogía ignaciana junto a los avances de la pedagogía, la psicología y la neurociencia. Hablar de participación y de la importancia del proceso para llegar a los resultados. Mirar alrededor y ver la necesidad de un nuevo espacio físico con una organización flexible y con un nuevo modelo de gestión del tiempo. Plantearse que los colegios que empujan esta iniciativa quieren formar personas conscientes, competentes, comprometidas y compasivas. Que asumen respecto a sus alumnas y alumnos una misión confesional de presentarles a Jesús y hablarles de Él, con experiencias de fe y compromiso.

Estamos hablando de cerca de 13.000 personas que conforman la comunidad educativa en torno a Jesuïtes Educació, la fundación que reúne a todos los centros de la Compañía de Jesús en Cataluña. Como señalaba Lluís Magriñà, que era provincial de Cataluña cuando en el año 2012 se puso en marcha el Horitzó 2020, "tenemos que ser agentes del cambio y de la transformación que buscamos. 


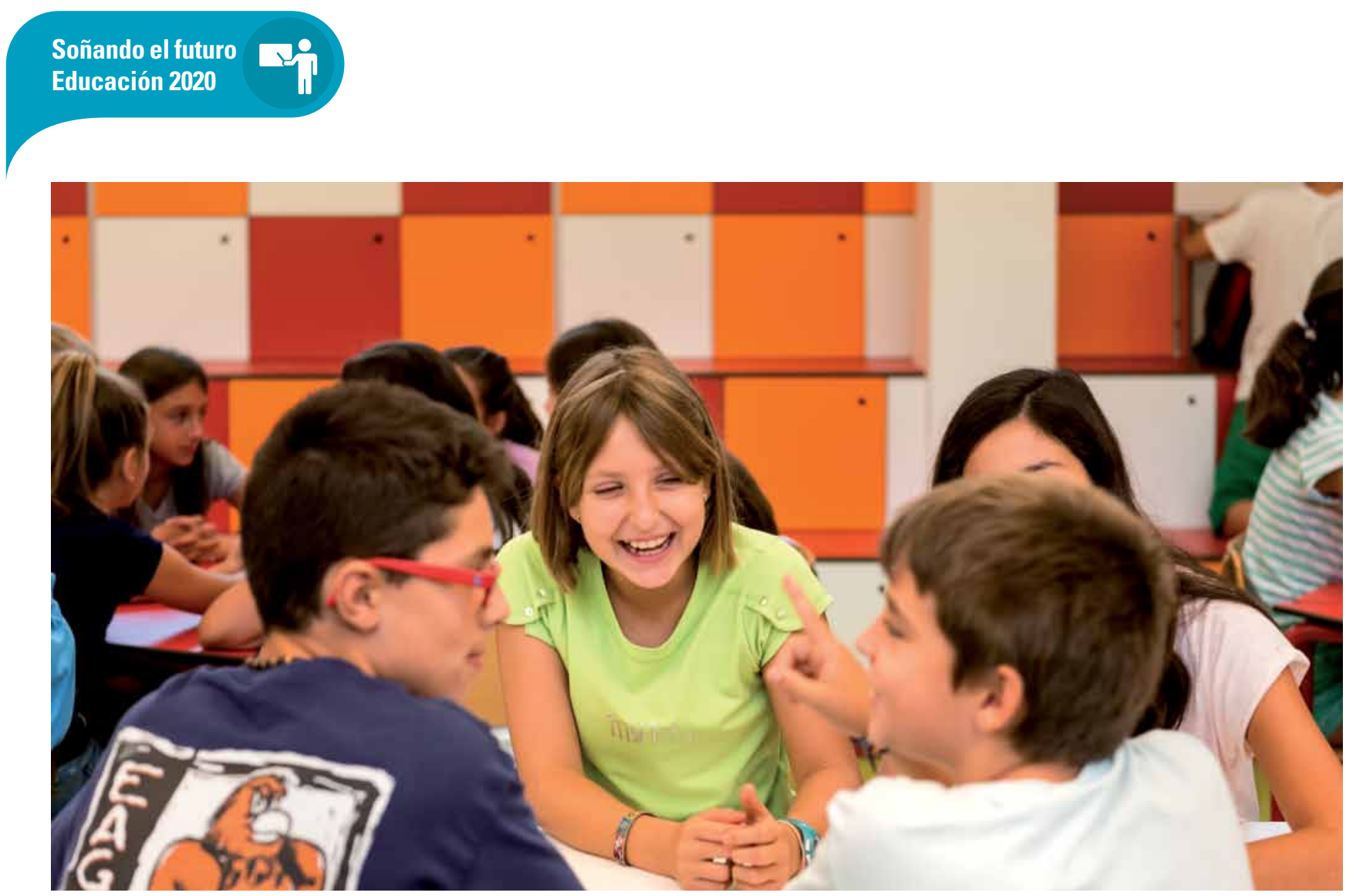

Tenemos las condiciones, no podemos dar un paso atrás. No es la hora de los indecisos. En caso de duda, hay que actuar. Es mejor equivocarse que no hacer nada, porque entonces sí que nos habremos equivocado".

$Y$ añade:

“No estamos ante un cambio de técnicas pedagógicas en nuestras escuelas, ni de mejorar la acción pastoral ni de llevar a cabo pequeños cambios. Estamos ante una trasformación profunda que haga sostenibles nuestras escuelas, que dé sentido a nuestra acción educativa y que sea ejemplar en la concreción del compromiso evangélico de la Compañía de Jesús".

Un año después, junio 2014, con motivo del final de su cargo como provincial remataba: "El Horitzó 2020 es fundamental, una nueva oportunidad para la evangelización. Tenemos que educar, formar y acompañar a los alumnos, ayudándolos a que encuentren su camino para construir su proyecto vital".

Llorenç Puig, nuevo delegado de los Jesuitas de Cataluña, que recibe el relevo de Magriñà, dice en junio de 2014: “Si no existiera Jesuïtes Educació se tendría que inventar. Tiene un papel imprescindible por su capacidad de liderazgo transformador y de impulso de la innovación para las escuelas jesuitas de Cataluña".

\section{Cuatro cuadernos, anatomía de un proyecto}

A primeros de marzo de 2015 Jesuïtes Educació sacó a la luz cuatro cuadernos (Transformando la educación) que rondan las 100 páginas cada uno, en los que se exponen las coordenadas fundamentales del Horitzó 2020. Están disponibles en su web (http://h2020.fje.edu/) y, articulados en epígrafes sencillos (40 consideraciones para enfocar el objetivo, 35 claves para preparar el terreno y propiciar el cambio, 37 metas como horizonte para soñar y 35 pasos que llevan a la acción, respectivamente), sintetizan sus propuestas para el cambio educativo que persiguen.

Casi 150 epígrafes que son una fuente directa para desentrañar los detalles de esta propuesta de cambio $y$, aunque es una tarea imposible sintetizarlos en este artículo, sí queremos hacer dos cosas: primera, animar a su consulta directa y a la lectura en función de los intereses de cada cual y de las circunstancias educativas de su entorno (el trabajo en el aula, la dirección de proyectos, la reflexión sobre las ideas fundamentales, la gestión de los centros, la innovación didáctica, etcétera). Segunda, destacar, como hacemos a continuación, algunos aspectos relevantes que pueden ayudar en la consulta de estos materiales. Vamos a ello. Escuchemos a los protagonistas.

\section{El objetivo}

"Ite inflamate omnia, Id y dad vida a todo"

San Ignacio

Manejamos modelos pedagógicos agotados, y necesitamos un nuevo sistema que impulse y facilite el cambio. La cultura educativa es la clave, pero los obstáculos son poderosos: la escuela es uno de los lugares en donde más se habla de trabajo en equipo y donde menos se practica. Educar 
tiene que significar la integración del arte de enseñar en un proceso más colectivo donde cada uno pueda encontrar su lugar dentro del equipo según sus aptitudes.

Hay que vincular la innovación a la tarea colectiva del profesorado, a la mejora de resultados y a la obtención y registro sistemáticos de los datos del proceso de enseñar y aprender que incluyan su impacto.

Tenemos que ver la escuela de una forma nueva: la creación de una verdadera red de escuelas que comparten un propósito de cambio profundo es un elemento clave (y previo) para preparar e iniciar la transformación de la educación.

Liderazgo pedagógico frente a liderazgo burocrático: la mejora de los aprendizajes de los alumnos y de los resultados e impacto del centro son la prioridad. El trabajo horizontal en red necesita un norte, una dirección que sea capaz de guiar las acciones que deban emprenderse. Más allá de las cuestiones administrativas, las directoras y los directores deberán crear las condiciones y los contextos para que los docentes puedan con un alto grado de autonomía hacer mejor su trabajo.

Ahora bien, aunque la transformación de la cultura educativa sea la clave, se traducirá en cambios concretos que necesitarán apoyo económico. Renovación, inversión y eficiencia parecen ser los vectores para esta nueva escuela. La participación del Estado y de las familias en un servicio que hoy día se concibe como gratuito, y la aportación de recursos en consonancia con las necesidades del nivel sociocultural, son imprescindibles para potenciar la mejora continua del proceso de enseñanza y aprendizaje.

¿Hacia dónde queremos ir? Cuando en Horitzó 2020 hablamos de proyecto estamos hablando de proyecto de futuro, de proyecto educativo de cambio, del horizonte que vislumbramos en los próximos años de transformación. Al mismo tiempo, definir el ideario y la misión de una escuela es el modo de transcender el plano individual y pasar al plano colectivo.

El proyecto educativo, que fue un concepto que se difundió con fuerza en España a finales de los setenta (a la par de las necesidades sociales y políticas del momento), se ha convertido con el paso de los años en un documento retórico, un formalismo que reclama la Administración, lejos muchas veces del devenir real de la propuesta educativa y metodológica del centro, vacío de contenidos pedagógicos.

Alumnos, educadores, familias, renovación de los profesionales o cambios físicos en el entor-

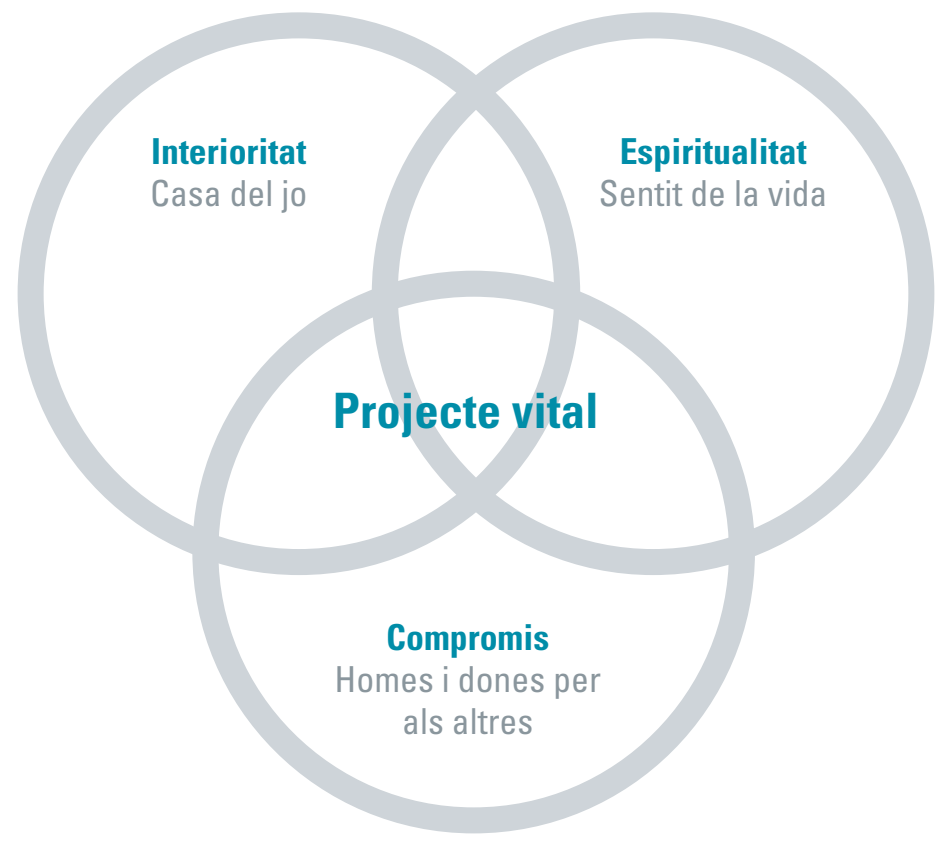

no escolar pueden concretarse de muy diferentes formas. Es un cambio sistémico, que dará lugar a un proyecto propio, capaz de concretar una experiencia de innovación educativa trasformadora y singular.

El currículo oficial, los conocimientos básicos, la transversalidad, la comprensión de la globalidad, el desarrollo de las competencias y, en fin, el respeto a los ritmos de los protagonistas de la escuela, han de formar parte de la fórmula que pongamos en práctica para acompañar con todos nuestros recursos el crecimiento de los alumnos.

Las 4C, o el modelo de persona que vertebra nuestro proyecto educativo: personas competentes, conscientes, compasivas y comprometidas. Un compromiso para educar, en colaboración con las familias, para que las alumnas y los alumnos descubran todo su potencial y desarrollen su proyecto vital y personal en los tiempos que les tocará vivir. El Horitzó 2020 reúne estas cuatro características en una quinta, denominada identidad propia y proyecto vital; en otras palabras, el descubrimiento de la vocación personal y la capacidad de liderar y conducir la propia vida.

\section{Por dónde empezar}

"Yo no enseño a mis alumnos, sólo les proporciono las condiciones adecuadas para que puedan aprender"

\section{Albert Einstein}

Para un cambio de estas dimensiones y, sobre todo, de esta profundidad, hay que asegurarse de que el propio proceso de cambio hace participar a 


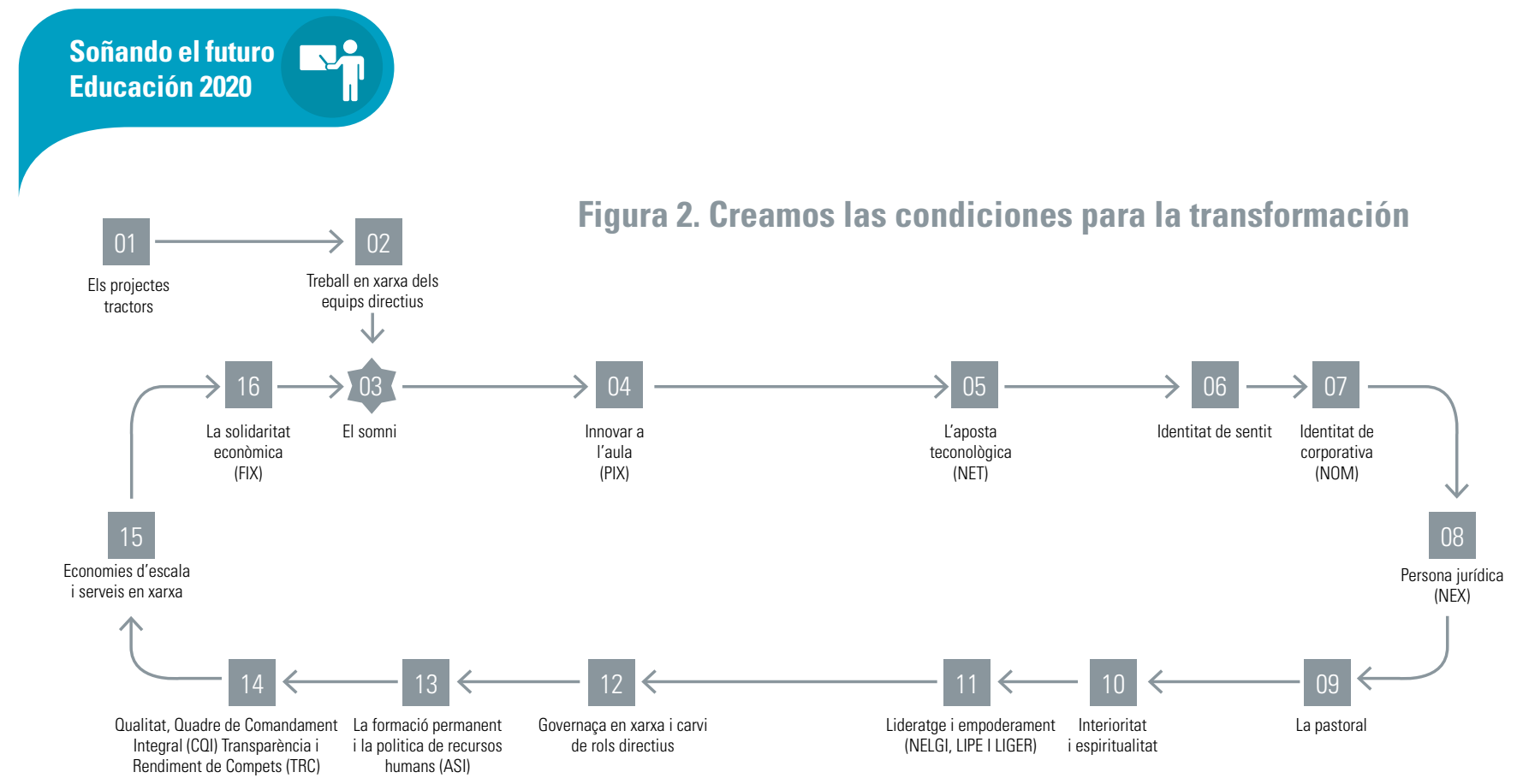

toda la comunidad educativa. Lo dice con rotundidad Xavier Aragay, director general de Jesuïtes Educació:

"Si tenemos como seña de identidad el compromiso con la escuela que queremos para el siglo XXI, hemos de crear las condiciones para que los profesionales, los alumnos y las familias puedan protagonizar esta transformación educativa. Es preciso preparar el terreno para la innovación, y no hacerlo sería como sembrar un campo sin haberlo arado nunca".

\section{Cuadro 1. No es una sopa de letras, son herramientas}

ע UBP. Unidades básicas de participación. Reuniones de maestros, educadores, alumnos, personal de administración y servicios, familias. Decenas de reuniones y mucho más de mil participantes para recoger ideas y propuestas.

ע AIP. Agentes impulsores de la participación. Más de un centenar de educadores con la tarea de motivar a todas las personas implicadas en las UBP.

ע Seminarios de reflexión y debate de Jesuïtes Educació. En palabras de Pep Menéndez, director adjunto de Jesuïtes Educació, son un ecosistema de pensamiento y debate colectivo que nos ayuda a profundizar en el qué y a establecer los cómo de nuestro futuro educativo. Misión, gobernanza, gestión y liderazgo del cambio son sus ejes organizadores.

У APA. Actividades de participación del alumnado. Adaptadas a cada edad, desde los niños y las niñas de P-3 hasta el Bachillerato y los ciclos formativos, dinámicas inéditas y muy preparadas, dirigidas por los tutores.

У SIPEI. Seminario internacional de pedagogía y espiritualidad ignaciana. A través de él, el Secretariado para la Educación de la Compañía de Jesús impulsa una reflexión sobre los retos y las oportunidades de la educación hoy en el mundo. Celebrado en noviembre de 2014 en Manresa, es la continuación del Coloquio Internacional de la Educación Secundaria Jesuita celebrado en Boston de 2012 y gira en torno a los cuatro ejes de la persona ya mencionados: conciencia, competencia, compasión y compromiso.

y MEG. Modelo estratégico de gestión. Nueva organización y nuevos espacios. El trabajo de los equipos directivos con la ayuda del técnico de gestión (TEG).
Buena idea, sin duda, pero lleva su tiempo. Podemos pensar que el Horitzó 2020 ha comenzado hace quince años..., o quizá unos siglos más atrás.

Sí, tiene unas raíces con más de 400 años de historia: desde los primeros trazos del jesuita segoviano Diego de Ledesma, hasta la redacción final de la Ratio Studiorum en 1599, este "plan de estudios" supone el primer currículo moderno, transnacional (en Europa y allí donde se fundaban colegios por todo el mundo), con una metodología pedagógica específica y una organización escolar propia. Potentes elementos comunes, con una concreción particular para cada escuela, sujeta en cada caso a una iniciativa específica en un contexto diferente: "no todo conviene a todos ni de la misma manera", en la conocida frase de la época. Habrían de pasar más de 300 años para que un nuevo documento refrescara esa visión común de los centros educativos de la Compañía de Jesús. Habrían de pasar más de 300 años para que un nuevo documento refrescara esa visión común de los centros educativos de la Compañía de Jesús. Esta renovación la inició el padre Arrupe al final de su mandato, aunque no se publicó hasta 1986 con el título Características de la educación de la Compañía de Jesús. Este texto que fue seguido en 1993, ambos bajo el mandato como General del padre Kolvenbach, por otro con propuestas metodológicas concretas titulado Paradigma pedagógico ignaciano. Son los documentos que han servido como referencia en las escuelas jesuitas de los últimos veinte años.

Recoger con un mínimo de detalle la "preparación del terreno" que precede al nacimiento del Horitzó 2020 exigiría otro artículo completo como éste. Así que nos vamos a conformar con cifrar los inicios de estos trabajos de renovación en el año 2000, cuando bajo la dirección de Lluís Magriñà se crea la Fundació Jesuïtes Educació. La reflexión estratégica, el trabajo en red, los planes estratégicos, el papel de los "proyectos tractores" (que además 


\section{Cuadro 2. No es una sopa de letras, son ideas} transformadoras..

de su finalidad propia a corto plazo sirven, más a fondo, para "arar el campo", para preparar esos terrenos de la transformación de las personas y de la cultura de la organización) y, en fin, toda una sopa de letras propia (que no podemos desmenuzar aquí, véase el cuadro adjunto que hemos elaborado para el Horitzó 2020) imposible de detallar.

0jo, una sopa con mucha miga, basta saborear con atención las siglas para, al menos, hacerse una idea de lo que llevan detrás: PEA (evangelización, personas y pedagogía en torno al proceso de enseñanza y aprendizaje); PIX (proyectos de innovación en red-xarxa en catalán- como radionet, legoPIX, smartphones en el aula, emprendimiento, huertos escolares o tabletas); NET, la apuesta tecnológica; NOM, una identidad corporativa que diese testimonio de la unidad en red $y$, al mismo tiempo, permitiese la expresión singular de cada proyecto; NEX (nueva estructura en red, pieza jurídica para compartir la misma titularidad, personalidad jurídica y NIF) que, aunque a primera vista pueda parecer burocracia legal, da un nuevo impulso al sentido de pertenencia y, por ejemplo, abre la puerta a que los educadores puedan cambiar de centro en el marco de su progresión profesional. Cambiar de escuela puede renovar, abrir la mente, romper rutinas, nos puede hacer salir, en definitiva, de la zona de confort, nos pone en marcha; CLIP (curso de liderazgo ignaciano de la pastoral), para reforzar y liderar una pastoral común basada en una red de equipos laicos; una apuesta por la pedagogía de la interioridad y la espiritualidad; estrategias de liderazgo y empoderamiento como NELGI (nueva estrategia y liderazgo de la gobernanza integral), LIPE (liderazgo pedagógico) o LIGE (liderazgo de gerentes); ASI (atracción, selección e incorporación) para articular la formación permanente y la política de recursos humanos con un sistema único y en red para los nuevos educadores de las ocho escuelas.

La lista parece interminable, pero el terreno que hay que preparar para la innovación es muy amplio y no nos resistimos a señalar aspectos tan importantes para la gestión de la calidad en las escuelas como un cuadro de mando integral (COI) 0 los mecanismos de transparencia y rendimiento de cuentas (TRC). Se completa sin duda con los sistemas de información y comunicación (SIC), gestión de personas (GEPE), economía y finanzas (ECOFIN), programa de nóminas, contabilidad y gestión académica, y mantenimiento de obras (MIO). En fin, cerramos esta larga lista al señalar que la solidari- y CETEI. Centro de Tecnologías Ituarte de la Fundación Juan XXIII. Centro de innovación educativa para la formación personal y profesional, con especial incidencia en las TIC y en el liderazgo tecnopedagógico. Ejercerá de supervisor, garantizando la recogida de datos, el tratamiento de la información, y los objetivos de observación, reflexión y evaluación.

$\checkmark$ PEA. Proceso de enseñanza y aprendizaje. El alumno como centro de una educación que le ayude a integrar y conducir su propia vida. Un proceso de transición de la enseñanza hacia el aprendizaje.

ע NEE. Nueva estrategia evangelizadora. La renovación del modo en el que situamos a Jesús en el centro de nuestras escuelas, para vincularlo al desarrollo del proyecto vital de cada alumno.

$\checkmark$ MEJE. Modelo educativo de Jesuïtes Educació, un modelo de modelos: educativo, pedagógico, de gestión y del espacio físico.

$\checkmark$ MENA. Modelo de enseñanza y aprendizaje. Con cuatro grandes ejes: 1. Los alumnos y el equipo docente como esqueleto del modelo. 2. Contenidos (conceptos, procedimientos y valores) y metodología (trabajo individual y en equipo). Interacción de los alumnos, los profesores y los contenidos. 4. Las familias, con una comunicación fluida y frecuente ente familia y escuela.

$\checkmark$ MCEFE. Modelo de cambio del espacio físico de las escuelas. Aulas más grandes, con más color y más luz, con mejor aislamiento acústico, con gradas, con mesas que permiten cambiar fácilmente la distribución del aula y trabajar por grupos, con sala de reuniones, con un espacio para los profesores que trabajan allí.

dad económica entre los centros tiene que avanzar y para ello se crea el fondo de inversión en red (FIX) que regula la aportación diferenciada de cada centro a los gastos comunes de la red según su nivel socioeconómico.

Mecanismos como los que acabamos de apuntar son los que han permitido lanzarse a la piscina, iniciar el camino, avanzar y retroceder para mejorar, evaluar el proceso y, desde luego, celebrar las metas y los éxitos para aumentar el caudal de energía individual y colectiva para continuar progresando. El segundo cuaderno termina con una docena de preguntas que permiten evaluar en qué grado se está preparado para seguir avanzando. Dicho de otra forma, desde el año 2000 se han ido incorporando de forma gradual respuestas a estas preguntas, respuestas afirmativas que permiten pasar con garantías a la siguiente fase del proceso.

Aquí no tenemos más espacio, así que solamente cabe insistir de nuevo en que lean este Cuaderno $n^{0} 2$ con calma y detalle, sobre todo si quieren captar que hacer camino es una cuestión de ideas, acuerdos y convicciones, sí, pero sobre todo de vivencias con emociones y sentimientos. Si estas comunidades educativas han introducido en su hablar diario palabras como "pix", "lipe" o "net", hemos de percatarnos de que están ampliando su lenguaje y, al hacerlo, 


\section{Cuadro 3. Vocabulario nuevo para nuevas ideas} educativas

ע NEI. Nueva etapa intermedia. Cuatro años, de $5^{\circ}$ de Primaria a $2^{\circ}$ de ESO, para responder con unidad y coherencia psicopedagógicas a la evolución natural de estas edades y potenciar las inteligencias múltiples de cada alumno.

У MOPI. Modelo pedagógico en la etapa infantil. En proceso inicial durante este curso 2014-2015 en los cursos de P-3 de cuatro escuelas de la red.

\ PIEP. Programa de incorporación a la experiencia piloto. Formación y acompañamiento para el cambio, que permita iniciar con éxito la experiencia piloto del nuevo modelo educativo. Cuatro bloques con un total de 450 horas de formación.

ע EAR. Equipos de alto rendimiento. Son unidades de alta calidad humana y profesional que aportan innovaciones concretas y experiencias de aprendizaje modélicas para la red. Parten de un gran compromiso e identificación con su propósito y cuentan con los recursos y la autonomía necesarios para llegar a su objetivo. vean a los distintos grupos participando, es tiempo bien empleado: en pocos minutos desfilan ante sus ojos padres y madres, alumnos, profesores y otros miembros del personal de los centros y de los equipos directivos; se oye lo que dicen, se palpa su interés por participar en este proceso, imágenes e ideas saltan delante de nosotros. No lo podemos resumir aquí, pero está a su disposición, no se lo pierdan.

Media docena de ámbitos de trabajo articulan este esfuerzo colectivo a lo largo de muchos meses: 1. Método, contenidos y valores. 2. Alumnos y profesorado. 3. Espacios educativos. 4. Tiempo y organización. 5. Tecnología y recursos. 6. Familias y entorno. Son a menudo, como en las UBP (unidades básicas de participación), dos horas muy productivas para escuchar e intercambiar ideas. Son un debate orientado, que no se queda en mera tertulia, que cuenta con el apoyo de los AIP (búsquelo en la lista...). Como señalaba en 2013 Lluís Magriñà: "Estoy muy orgulloso del proceso de reflexión y participación que estamos desarrollando. Conozco muchos lugares del mundo y no había visto una iniciativa tan valiente y decidida como ésta".

El Horitzó 2020 aterriza en 17 ideas clave para la escuela que queremos. En 10 características para la persona que estamos buscando. No es que los números importen, es la forma de comunicar las ideas y de mover a la reflexión sobre ellas. El Horitzó 2020 propone un nuevo modelo educativo (MEJE), un "modelo de modelos": educativo, pedagógico, de gestión y del espacio físico. Así lo señala el padre general Adolfo Nicolás:

\footnotetext{
“Los jesuitas estamos en la educación porque desde el comienzo la gran preocupación de San Ignacio y de sus compañeros y seguidores es el crecimiento y la transformación de la persona. No estamos aquí sólo para enseñar o comunicar algunas técnicas de éxito, sino para acompañar, desde muy pequeñas, a las personas en su crecimiento, que es lo más importante que podemos hacer en la vida".
}

El nuevo modelo de enseñanza y aprendizaje pone el foco en siete ámbitos competenciales: comprensión lectora, expresión oral y escrita, resolución de problemas, habilidades digitales, interioridad, aprender a aprender y valores sociales. Con una metodología que potencia el aprendizaje por descubrimiento, que incentiva la autonomía de los alumnos, la creatividad, la observación, el razonamiento y la práctica científica. Forman parte 


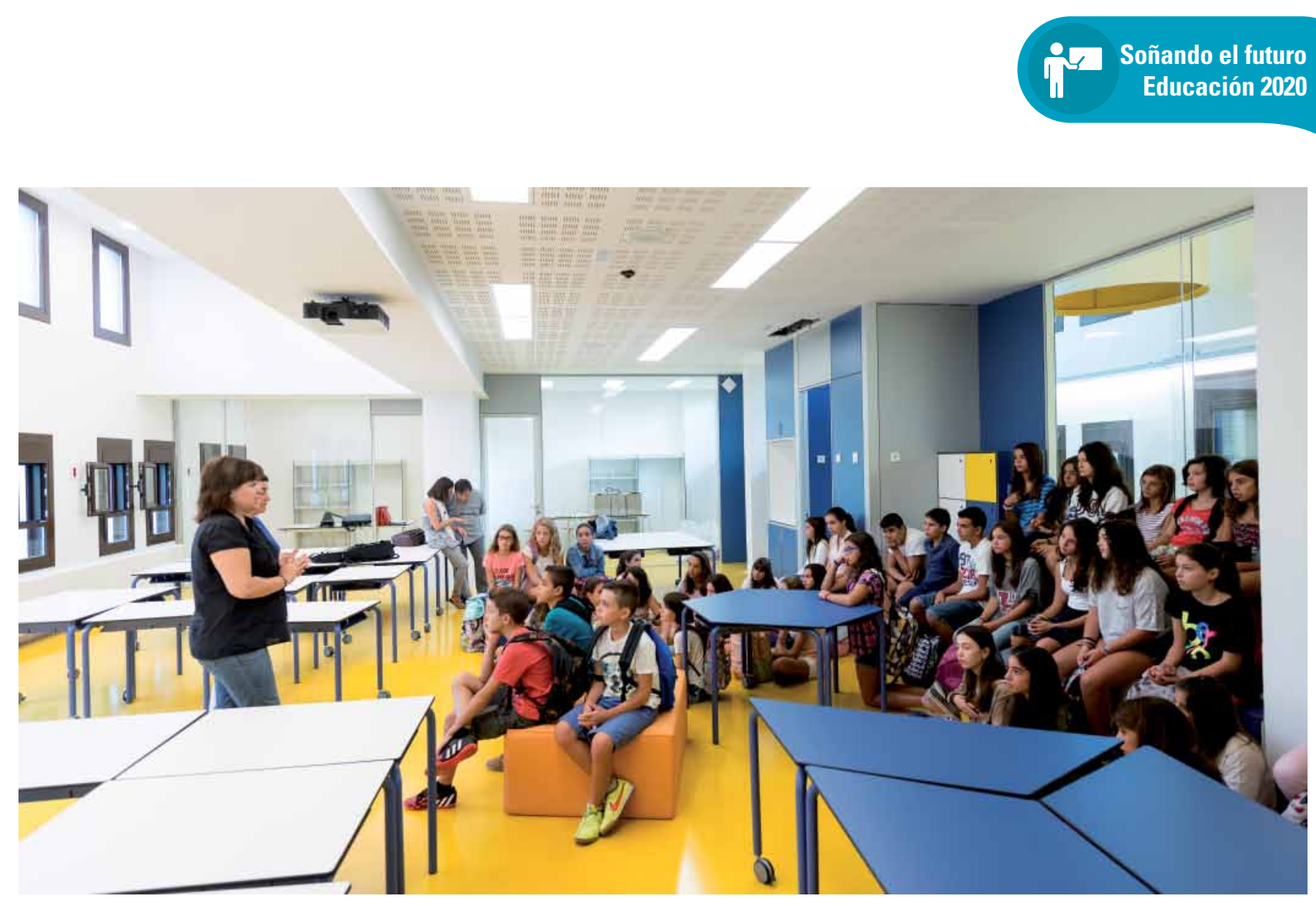

del MENA el aprendizaje por proyectos, la resolución de problemas y el aprendizaje cooperativo.

En fin, formular el Horitzó 2020 ha supuesto sobre todo la activación de la red, de la vocación y de los sentimientos y las emociones de todos los participantes. Toda la comunidad trabajando por el cambio, esforzándose por sumar iniciativas, debatiendo, implicándose con una sonrisa. Una ola de emociones por el cambio.

\section{¡Acción! \\ “No pido que me expliquéis por qué es imposible, sino que descubráis qué hay que hacer para que sea posible"}

Jacques Steffens, empresario

El curso 2014-2015 ha sido el comienzo, en varias escuelas de la red, de las experiencias piloto. Es la hora del cómo: ¿en qué se concreta nuestro sueño compartido? Se ha profundizado en el trabajo de los seminarios (para ello se han definido grupos que, por encima de $2^{\circ}$ de Primaria, abarcan cada uno a tres cursos) y se ha insistido tanto en la necesidad del retorno de las ideas de cada sesión hacia los interesados y hacia el conjunto, como en los marcos sucesivos que la puesta en marcha con carácter piloto de este cambio tienen como referencia. Son experiencias, no experimentos, y toca vivirlas, en los dos cursos 2014-2016, con interés, rigor y profesionalidad para que se conviertan en lo más ricas y útiles posibles.

Dos experiencias: se ha comenzado con la Educación Infantil (MOPI), en el curso de 4 años, y con la Nueva Etapa Intermedia (NEl, de $5^{\circ}$ de Primaria a $2^{\circ}$ de
ESO, lo que supone una nueva manera de articular las etapas educativas, con la que se quiere dar respuesta a la evolución natural de unidad y coherencia psicopedagógica de estas edades). Los centros pensaron sobre esta fase y solicitaron o no en cada caso su participación en ellas. Como resultado, en septiembre de 2014 la NEI fue realidad en tres centros (615 alumnos, se ha comenzado en $5^{\circ}$ y en $1^{\circ}$ de ESO, para pasar el año que viene a $6^{\circ}$ y $2^{\circ}$ ), y el MOPI en cuatro (225 alumnos). Más de ochocientos alumnos junto a un centenar de educadores, entre tutores, especialistas, gestores y otros profesionales (el apoyo del CETEl en este proceso es esencial) que ponen en práctica otro proceso de enseñanza y aprendizaje.

Trabajo por proyectos, reuniones frecuentes con los padres, inserción del inglés en Infantil, tiempo flexible, recursos digitales, evaluación tanto de resultados como de procesos (con autoevaluación y coevaluación), integración de los valores... Una larga lista de elementos que forman parte de esta puesta en práctica, pero que no alcanzarán su significado si no se apoyan en la formación del profesorado. Por eso, desde noviembre de 2013 se pone en marcha un programa de incorporación a la experiencia piloto (PIEP) en el marco del cual se han seleccionado a 33 profesionales de las ocho escuelas que, junto con el equipo directivo de la NEI, configuran el primer equipo del PIEP. Estos 33 educadores vivirán los dos años de la experiencia piloto en uno de los tres centros de la NEI y después siete de ellos (que cambian de centro) regresarán a su escuela para transmitir los aprendizajes al equipo docente y directivo. Seis bloques de distinta duración, intensidad y frecuencia, que 


\section{Cuadro 4. Dónde hemos empezado}

Jesuïtes Educació está formada por una red de ocho escuelas: seis en Barcelona, una en L'Hospitalet, otra en Raimat (Lleida) y dos Fundaciones adheridas.

y Centre d'Estudis Joan XXIII (Bellvitge, Hospitalet de Llobregat)

$\searrow$ Col.legi Casp, Sagrat Cor de Jesús (Eixample, Barcelona)

$\searrow$ Col-legi Claver (Raimat-Lleida)

ע Col-legi Sant Estanislau de Kostka (Gràcia, Barcelona)

\ Col.legi Sant Pere Claver (Poble Sec, Barcelona)

ע Escola Tècnica Professional del Clot (Clot, Barcelona)

ע Sant Ignasi-Sarrià (Sarrià, Barcelona)

ע Sant Gervasi-Escola Infant Jesús (Barcelona)

ע Fundació Carles Blanch (Badalona)

У Fundació La Salut Alta (Badalona)

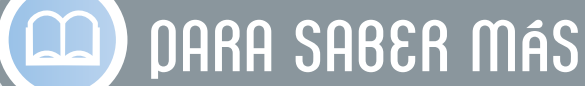

María Acaso. 2013. rEDUvolution. Barcelona: Espasa.

Un inicio de revolución educativa que parte de considerar que lo que se enseña no es lo que los alumnos aprenden. Que deben cambiarse las dinámicas de poder. Que aprender se da en cualquier lugar habitado. Que debe transitarse del simulacro a aprender viviendo experiencias. Que evaluar debe dejar paso a investigar.

Hay que abandonar las inercias de un paradigma educativo anclado y más cercano al siglo XIX y a la producción industrial para sumergirse totalmente en las dinámicas del siglo XXI y conseguir que el aprendizaje, por fin, suceda de una vez.

Sebastí́n Barajas. 2013. Aprender es hacer. Barcelona: Ediciones Invisibles.

A partir de los más recientes avances en ciencias cognitivas y siguiendo a Roger Schank, el libro presenta una clara propuesta de cómo adaptar el sistema educativo al siglo XXI. Se ofrece una alternativa contrastada el SCC (Story Centered Curriculum), una arquitectura orientada al desarrollo de programas educativos en los que los alumnos adoptan un papel activo y viven experiencias de factura similar a las que se encontrarán en la vida profesional que desempeñen.

David Perkins. 1995. La escuela inteligente. Barcelona: GEDISA.

Escuelas inteligentes son las que se mantienen atentas a todo posible progreso en el campo de la enseñanza y el aprendizaje. La escuela inteligente debe poseer tres características: Estar informada, ser dinámica y ser reflexiva.

Una escuela será inteligente en la medida que logremos pasar del adiestramiento de la memoria a la educación de la mente. La intención es clara: que los estudiantes no sólo conozcan, sino que piensen a partir de lo que conocen.

ElISENDA Soriguera. 2013. El camino del sueño: hacia el Horitzó 2020. "El relato". Barcelona: Fundación JE.

Reflexiones breves y a pie de obra durante el proceso de gestación del Horitzó 2020. 52 páginas de vivencias y anécdotas escritas por una observadora independiente. Disponible en: http://h2020.fje.edu/relat/ alcanzan más de 450 horas de formación, articulan este programa, que fomenta el trabajo en equipo y es, en suma, una invitación a la transformación personal para trabajar al servicio de los demás.

El cambio del espacio físico (MCEFE) es esencial, los arquitectos entran en juego y, tras las obras realizadas en el verano del 2014, los resultados ya son visibles. Esto no es gratis, pero un buen proyecto y una buena gestión económica del conjunto de los centros de la red son una bandera ilusionante para atraer financiación.

Una estrategia de comunicación amplia y variada (que además de los materiales disponibles en la web incluye las actividades realizadas con y por los propios alumnos, los vídeos, el cómic, los periódicos anuales del Horitzó 2020) permite recoger opiniones y logra que la red de escuelas esté abierta a las personas que se acercan con curiosidad y ganas de saber qué estamos haciendo y, sobre todo, cómo lo estamos haciendo.

\section{Un fuego que enciende otros fuegos}

Terminamos por hoy. Estos programas de formación (PIEP) han llevado a constituir equipos de alto rendimiento que cambian juntos y que van más allá del marco particular de cada escuela. Equipos capaces de innovar disruptivamente, de salir de la zona conocida, de ir más allá de lo que hacemos siempre, de las preguntas y respuestas habituales. Equipos con vocación, es decir, que descubren el regalo de ofrecer una aportación singular a la sociedad. Las personas conectadas con su vocación actúan desde su voluntad de servicio. Hagan lo que hagan, no lo hacen por su satisfacción personal, sino para cumplir la misión que les ha sido encargada.

Decía Jorge Wagensberg, cuando era director del Museo de la Ciencia de Barcelona, "cambiar de respuesta es evolución; cambiar de pregunta es revolución". Después de leer estas páginas. ... ¿ ¿les suena? •

\section{(2) \\ Horitzó 2020, innovación educativa, cambio educativo, pedagogía ignaciana, liderazgo pedagógico.}

Este artículo fue solicitado por PADRES y MAESTROS en diciembre de 2014, revisado y aceptado en marzo de 2015. 